\title{
LA OBRA DE PÍO DEL RÍO HORTEGA Y SUS CONSECUENCIAS EN LA NEUROPATOLOGÍA
}

\author{
Juliana Fariña González \\ Catedrática de Anatomia Patológica. Universidad Complutense. Madrid \\ Presidenta del Colegio Oficial de Médicos de la Comunidad de Madrid \\ Julio Escalona Zapata \\ Ex Profesor Jefe del Servicio de Anatomia Patológica. Hospital General Universitario Gregorio \\ Marañon. Madrid. \\ Profesor Titular de Anatomía Patológica. Universidad Complutense. Madrid
}

ABSTRACT The article summarizes the discoveries of Pio del Rio Hortega in the normal histology of nervous system and their subsequent influence in the neuropathology studies of brain tumours: gliomas and paragliomas.

KEY WORDS Histology of nervous system. Micrographic techniques. Microglia and oloigodendroglia. Neuropathology. Brain tumours. Gliomas and paragliomas.
RESUMEN El artículo repasa los descubrimientos de Río Hortega en la histología normal del sistema nervioso y su posterior repercusión en el estudio anatomopatológico de los tumores cerebrales: gliomas y paragliomas.

PALABRAS CLAVE Histología del sistema nervioso. Técnicas micrográficas. Microglía y oligondendroglía. Neuropatología. Tumores cerebrales. Gliomas y paragliomas.

\section{Antecedentes}

Con anterioridad a 1920 en que Río Hortega describiera la microglia y la oligodendroglía, la celularidad del Sistema Nervioso Central incluia dos tipos de célula. El primero, estudiado exhaustivamente por Cajal, son las neuronas, El segundo elemento vendría dado por la neuroglía de la que se distinguian dos subformas; la neuroglía fibrosa, propia de la sustencia blanca, provista de escaso citoplasma somatico y largas prolongaciones y la neuroglia protoplásmica de prolongaciones más cortas y gruesas. Hoy dia, gracias a la microscopia electrónica sabemos que estos dos pretendidos tipos celulares son una única celula y que las variantes protoplásmica y fibrosa de neuroglia, no dependen más que del mayor o menor contenido en gliofibrillas, más abundantes en la neuroglia de la sustancia blanca y menos en la neuroglia de la sustencia gris.

Además de estos dos tipos celulares -las neuronas y la neuroglia- existian en el cerebro numerosos elementos de nucleo redondo en unos casos y ovalado en otros, pero constantemente desprovistos de expansiones demostrables con los métodos clásicos, que fueron llamadas células apolares (Cajal), células de reserva (Schaper), etc. Este tercer elemento que mereció un trabajo especialmente dedicado a este tema, por Río Hortega ${ }^{1}$ es el que estaba reservado a este autor, cuyos trabajos, describiendo dos variedades celulares, ambas provistas de expansiones, han persistido hasta la actualidad. Se trata de la Microglia y de la Oligodendroglía.

En alguna ocasión hemos afirmado que parece como si Achucarro y Rio Hortega fueran la misma persona encarnada en dos vidas sucesivas. Efectivamente, Achucarro fue el primer español interesado en el estudio de las células intersticiales del Sistema Nervioso Central, dedicado hasta entonces, por la gran influencia de Cajal, al estudio de las neuronas y sus conexiones. Los métodos de impregnación con óxido de plata tras un mordentaje con tanino ${ }^{2}$ permitieron ahondar en la textura de la neuroglia ya descrita exhaustivamente por Weigert con su método a base de anilinas y por Cajal con la técnica del oro-sublimado. El método de Achucarro logro profundizar en las reacciones patológicas de la neuroglia, describiéndose sus alteraciones en la demencia paralitica, en el reblandecimiento cerebral asi como la glitectómica de la corteza cerebral. Un aspecto que seria aclarado mas tarde por Río Hortega, fue la naturaleza de los llamados cuerpos en bastoncito de la corteza cerebral. 
Muerto tempranamente Achucarro, su discípulo Río Hortega, modifico el método tanoargéntico, publicando cuatro modificaciones que sin dar por resultado unas imágenes decisivas si aportaron una mayor regularidad ante la variabilidad de la técnica original ${ }^{3}$. La consecuencia final de esos trabajos fue la introducción del carbonato de plata, reactivo mucho mas seguro y versátil que el oxido de plata, y que

Figura 1. Busto en bronce de Pío-Hortega. Museo de Valladolid

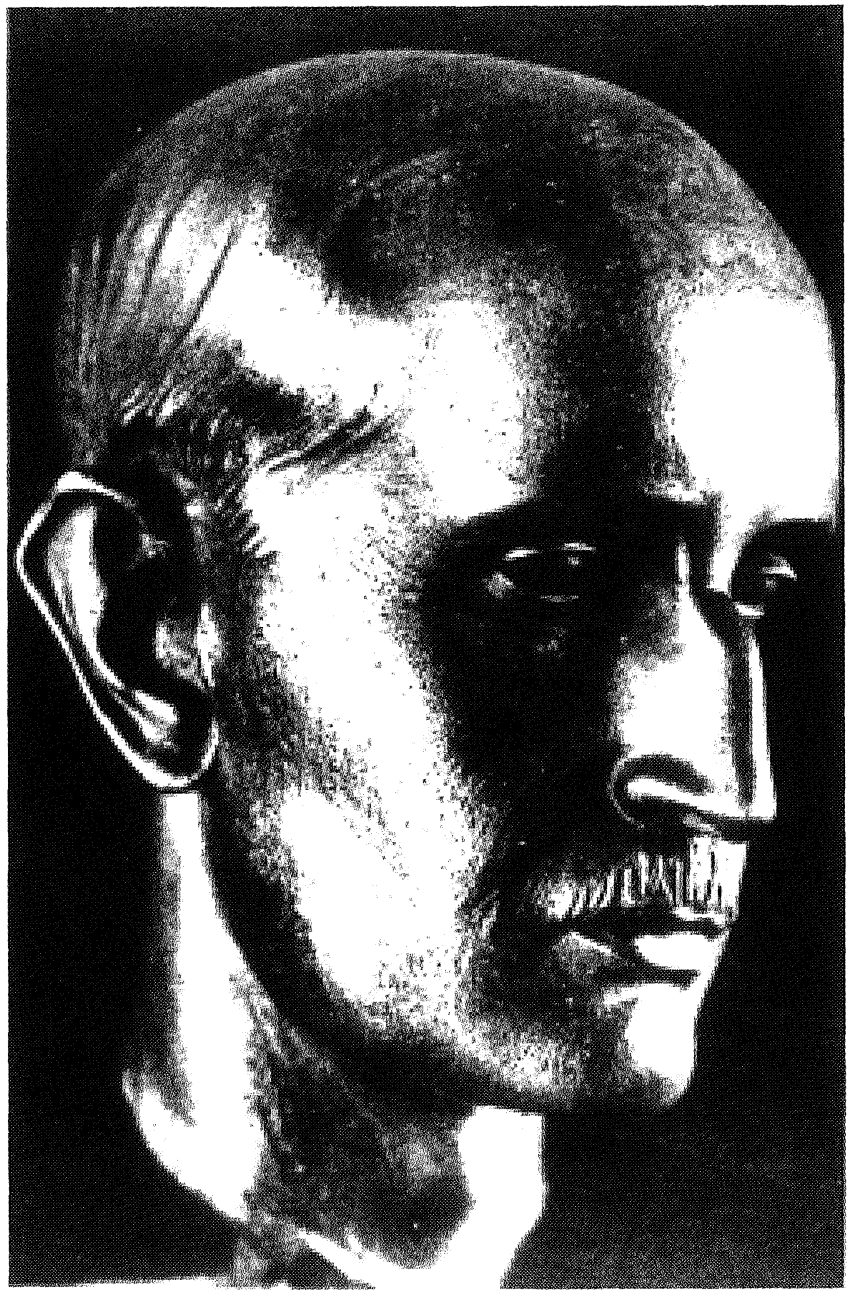

resulta de la precipitación del nitrato de plata con carbonato sódico o lítico según los casos, con ulterior disolución del precipitado con amoniaco ${ }^{4}$. De este modo, las irregularidades del método original de Achucarro quedaron obviadas y permitieron a Río Hortega abordar con éxito el problema de la naturaleza del llamado tercer elemento de los centros nerviosos.
Figura 2. Primera página del trabajo original

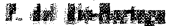

LO DUE DERE WNERDFAE POH TEDOED F1.6ENTO WE LOS CENTEOS NERVIOSOS

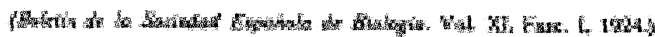


FiguRA 3. Primera página

del trabajo original

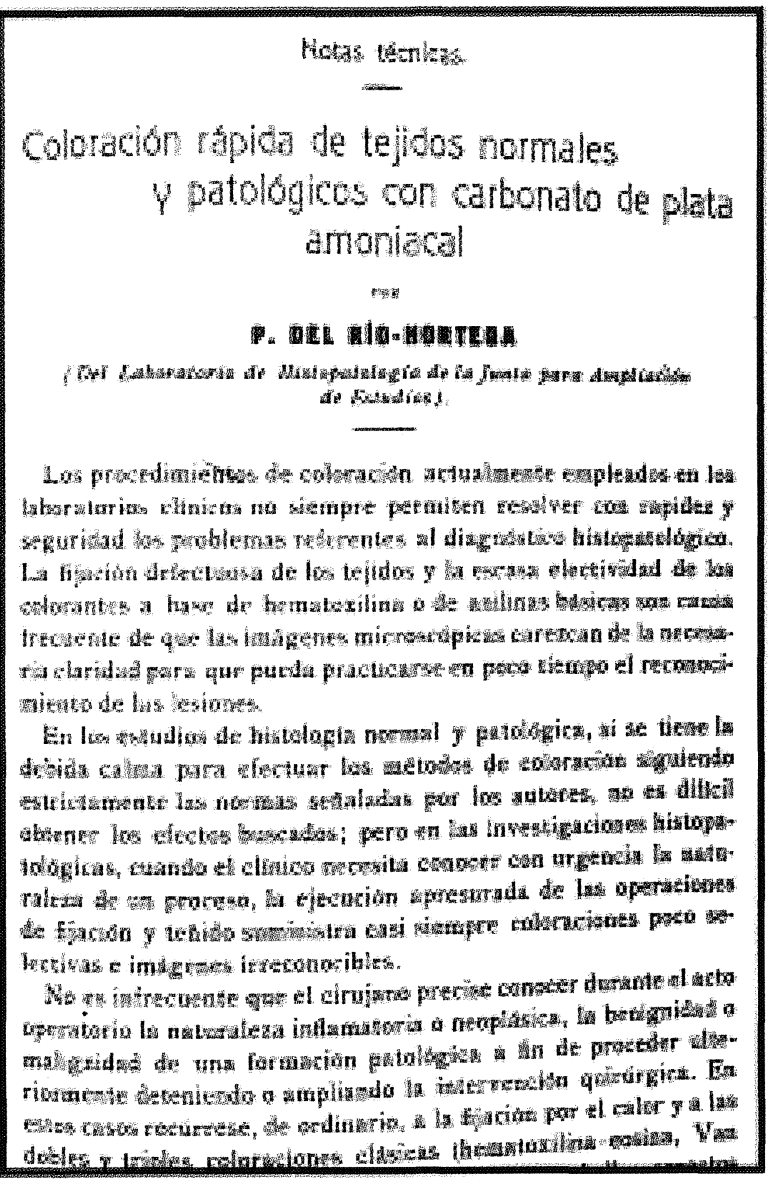

\section{La dependencia de la técnica micrográfica}

Realmente, buena parte de los avances de la Histología normal y patológica están basados en la técnica micrográfica. Dejando en lugar preferente la simple hematoxilina-eosina ${ }^{5}$ los tricrómicos han permitido separar los haces de músculo liso y las bandas de colágena y, en el caso del método de Masson, demostrar los humos propios de las glomerulonefritis. Las enfermedades desmielinizantes, son herencia del método, tantas veces modificado, de Weigert para la mielina. El empleo de los métodos de Cajal y Golgi fue el responsable de los grandes avances de la Neuroanatomía de la primera mitad del siglo XX.

Incluso, los nuevos conocimientos posteriores a 1950, se deben a la utilización de técnicas sucesivamente mas fia-
FIGURA 4. Primera página

del trabajo original

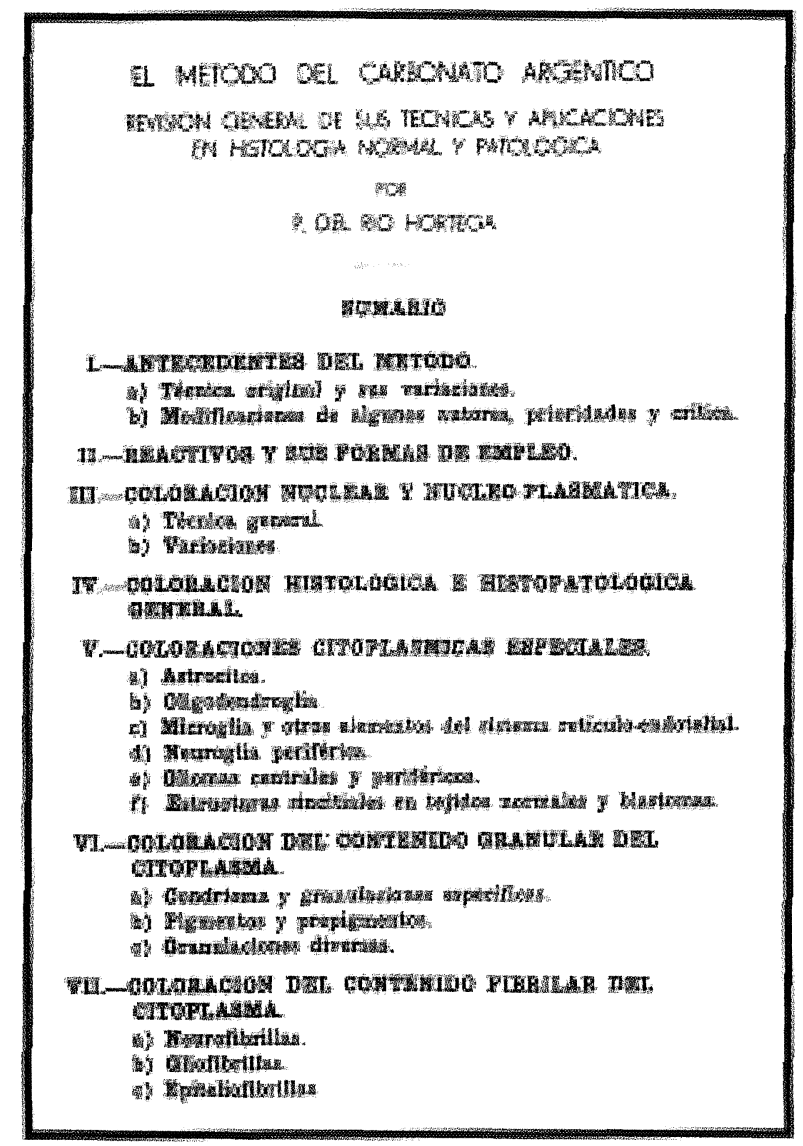

bles: la microscopia electrónica, el cultivo de tejidos, la inmunohistoquímica $y$, actualmente, la biología molecular.

En el caso de Río Hortega, la introducción de su reactivo -el carbonato de plata- fue decisiva para demostrar que las células apolares no lo eran realmente, sino que poseín expansiones no visibles con los métodos anteriores. Todo el edificio de la microglia y oligodendroglía en estado normal y patológico se asienta en estos dos métodos que solo han sido igualados 60 años mas tarde por las técnicas inmunohistoquímicas en el caso de la microglía CD68). Respecto a la oligodendroglía, aun se esta esperando una técnica que sea capaz de identificarla con carácter especifico. Incluso los tumores del Sistema Nervioso se han beneficiado de dichos métodos al ser los primeros que permitieron demostrar el citoplasma de las células neuroglicas tumorales ${ }^{6}$. 


\section{La microglía}

La existencia de un tipo celular de origen mesenquimal en el Sistema Nervioso Central habia sido entrevista por Roberston en $1900^{7}$, gracias a un método a base de sales de platino, asi como por Capobianco en $1901^{8}$ y por Hatai en $(1902)^{9}$. Dentro de este concepto de mesoglia de Robertson se englobaban todas las células de nucleo redondo $u$ oval con citoplasma invisible 0 ameboide, carentes de prolongaciones que habian sido consideradas por Cajal como el utercer elementon de los centros nerviosos ${ }^{1}$. Los elementos descritos por Nissl como células en bastoncito, representarian estos elementos o células neurogliales en actividad.

La tipificación exacta de las células del "tercer elemento" tuvo lugar entre 1920 y 1921, en que Río Hortega describió

FIgURA 5. Primera página

del trabajo original

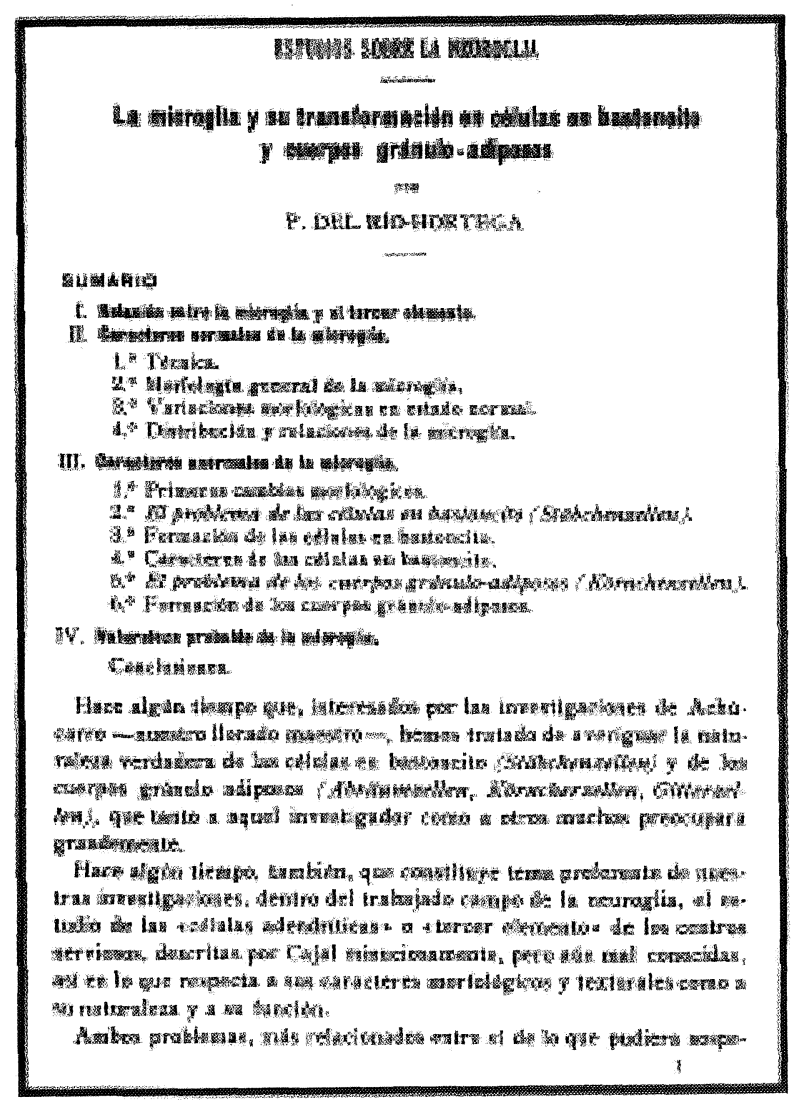

sus caracteristicas morfológicas en estado normal, su evolución para formar las llamadas células en bastoncito y en corpúsculos granuloadiposos, su origen mesodérmico y su distribución a lo largo de los centros nerviosos ${ }^{10,11,12}$.

Así, Río Hortega describió el origen tardio de la microglía, que penetra en el cerebro a través de piamadre de las telas coroideas superior e inferior. Desde estos puntos, las células mesenquimales se distribuyen por la corteza cerebral convirtiéndose en elementos de núcleo alargado, provistos de un citoplasma ricamente ramificado después de un trayecto relativamente perinuclear corto y casi siempre opositopolar. Solamente, cuando entran en actividad, estas células se transforman en células en bastoncito o células redondas o panzudas repletas de material fagcitado en el citoplasma; los cuerpos granuloadiposos.

FiguRA 6. Primera página del trabajo original

\section{El bencer foldonterto de los centros narviowos}

\section{Histogénesis y evolución normal; exodo y distribución regional de la microglia ${ }^{\text {th }}$}

\author{
The \\ *. Fin Hon

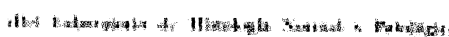

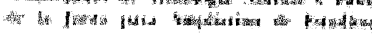

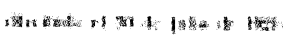

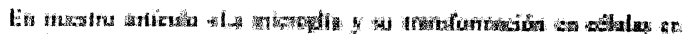

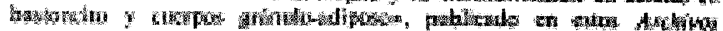

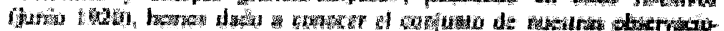

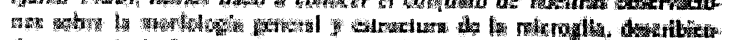

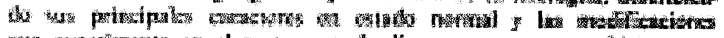

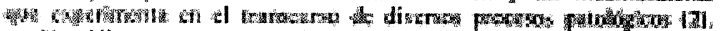

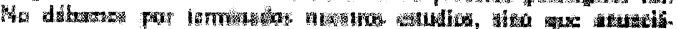

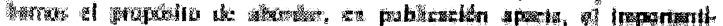

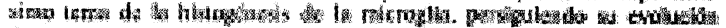

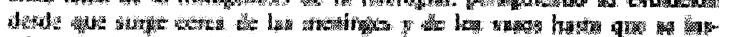

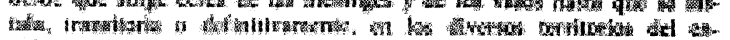
orks.

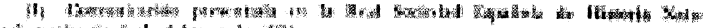

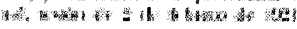

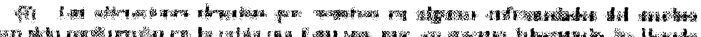
(1)

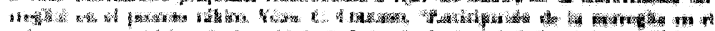

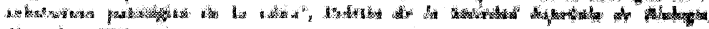

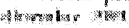


FiguRA 7. Imagen original del trabajo "La microglia y su transformación en células en bastoncito y corpusculos granuloadiposos" (1920)

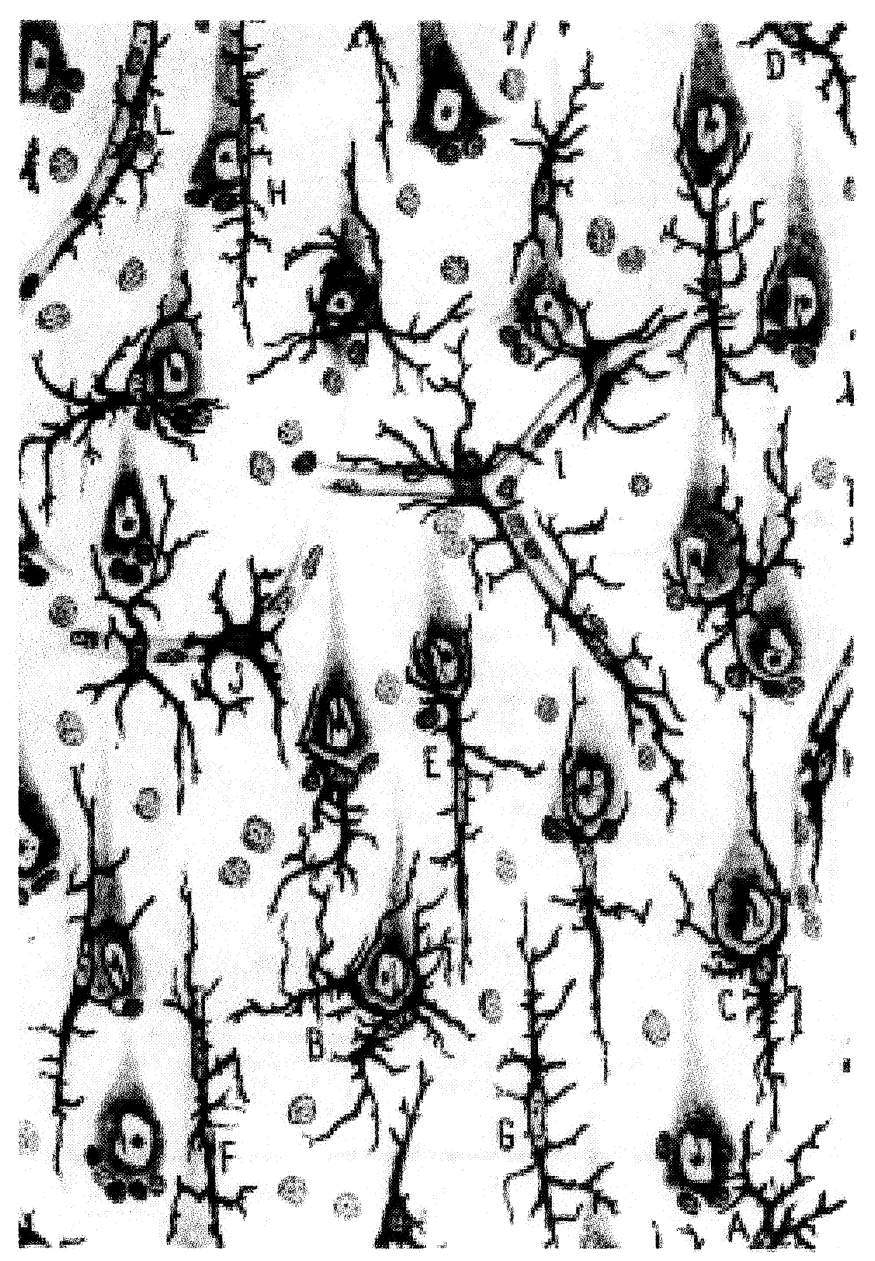

Contra estos puntos de vista no han faltado autores que se han pronunciado en sentido contrario, defendiendo su origen ectodermico. Buena parte de ellos han sido defensores de la teoria sincitial del Sistema Nervioso Central, por lo que actualmente han perdido toda su vigencia.

Por otra parte Fujita y Kitamura ${ }^{13}$, desarrollaron un modelo experimental por medio de la autorradiografia, demostrando el origen sanguineo del componente celular inflamatorio en los centros nerviosos. El fenomeno no tiene en si mayor importancia, no siendo más que la constatacion de un hecho habitual en el resto de los organos de la economia: la llegada al foco inflamatorio de elementos de origen sanguineo. El problema radica que en el mismo trabajo se niega la entrada tardia de la microglia en el neuroeje y se afirma su naturaleza ectodermica a partir del glioblasto. En nuestra opinion, y de acuerdo con opiniones expresadas a titulo personal por De Castro y Polak, el problema reside en que los autores japoneses no fueron capaces de teñir la microglia de los centros nerviosos en estado normal. La pretensión por parte de algunos de sus seguidores de que los conejos utilizados por Río Hortega no procedian de criaderos controlados y que todos ellos sufririan una encefalitis leve $y$, por lo tanto su microglía estaría activada, no pasa de ser una opinion gratuita si no es que es una pura anécdota.

De este modo, estos postulados han ido perdiendo valor hasta caer en el olvido.

\section{La oligodendroglía}

A pesar de que los términos células de Hortega, Hortegasche Zellen, Hortega'cells se refieran a la microglia, el descubrimiento de un nuevo tipo célula aun no descrito, corresponde a la oligodendroglia. En efecto, dentro de las células pretendidamente apolares del tercer elemento de los centros nerviosos, existe un tipo celular muy abundante, de núcleo redondo de aspecto linfocitoide, desprovisto de prolongaciones visibles con los métodos habituales. Este tipo de célula, tan abundante en la sustancia blanca, no podia pasar desapercibido, de modo que fue considerado como glía apolar mas o menos relacionada con el fenómeno de la neuro o pseudoneuronofagia (Cajal) o como células de reserva (Schaper) etc.

A partir de 1921, Río Hortega describe estas células como glia interfascicular o como glía de escasas radiaciones logrando demostrarla con dos métodos: el carbonato de plata y una variante del método de Golgi sin osmio. Con arreglo a sus descripciones la oligodendroglia se caracteriza por: A) Su abundancia en los centros nerviosos, especialmente en forma de hileras mal definidas en la sustancia blanca y de agrupaciones alrededor de las neuronas en la sustancia gris: B) Núcleo redondo; C) Expansiones escasas, finas o debilmente granulares con granulaciones (gliosomas) en el interior del citoplasma y D) Disposición enrollada alrededor de las fibras nerviosas, tan abundantes en la sustancia blanca $14,15,16$.

Tras estos trabajos, autores como Penfield ${ }^{17}$ Metz y Spatz ${ }^{18}$ aceptan los postulados de Río Hortega y mas tarde López Enríquez ${ }^{19}$ describe la oligodendroglia de las vías ópticas y Marchesani en ${ }^{20}$ la de la retina. 
FIgURA 8. Primera página

del trabajo original

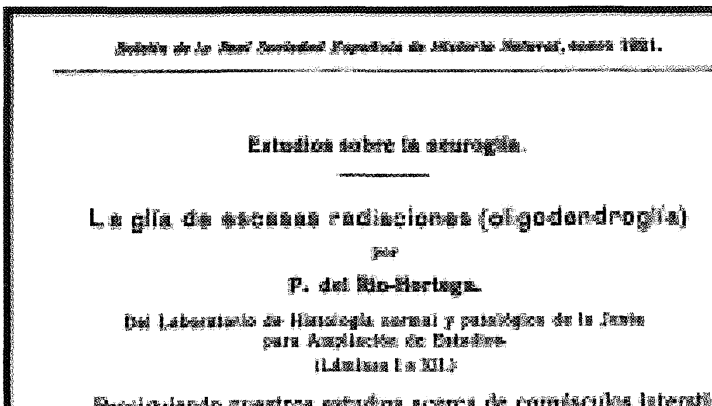

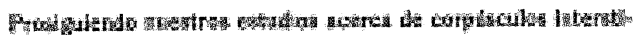

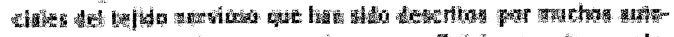

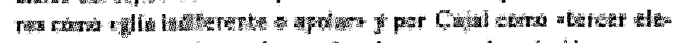

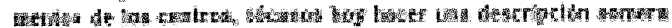

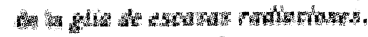

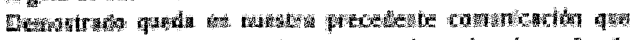

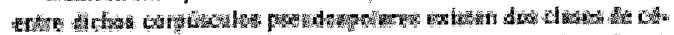

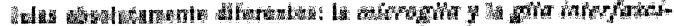

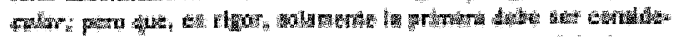

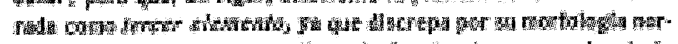

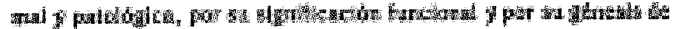

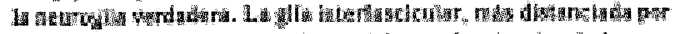

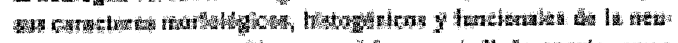

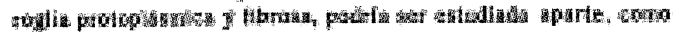

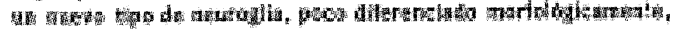

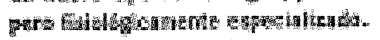

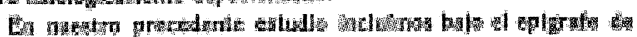

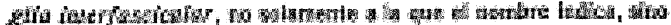

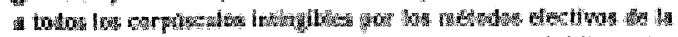

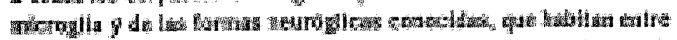

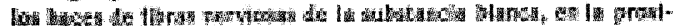

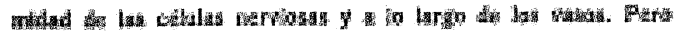

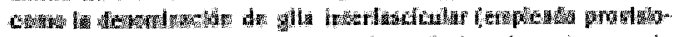
no

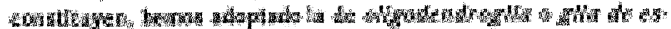

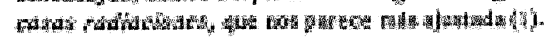

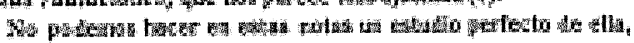

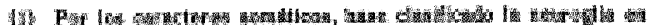

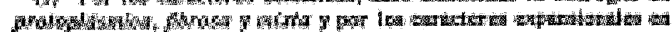

Un aspecto frecuentemente olvidado es la existencia de cuatro tipos de oligodendroglia, descritos en el ultimo trabajo de Rio Hortega ${ }^{16}$. En el, se hace hincapié en que, además de la morfología clásicas, que el llamo tipo I o de Roberson, existen formas de oligodendroglioma mas complejas, como los tipos II y III que Río Hortega Ilamo de Cajal y de Paladino, así como un tipo IV muy en relación con las fibras mielínicas. La existencia de estos subtipos explicaría la complejidad celular del oligodendroglioma cuando se estudia con métodos especiales.

La gran importancia de los trabajos de Río Hortega sobre la oligodendroglia reside en haber estudiado el tema de forma exhaustiva. No solo describió sus caracteristicas
FIGURA 9. Primera página

del trabajo original

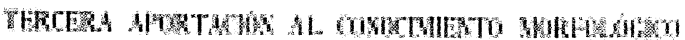

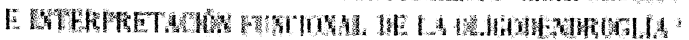

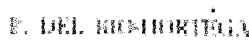

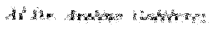

Eamanto,

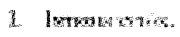

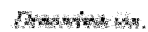

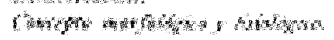

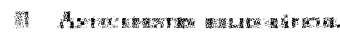

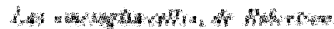

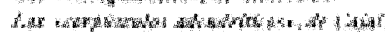

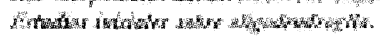

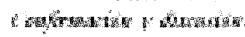

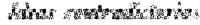

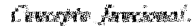

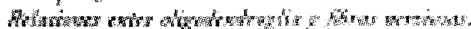

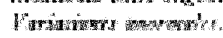

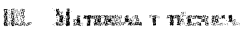

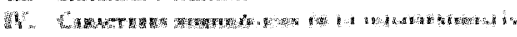

rians.

3.t.

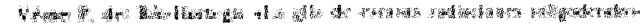

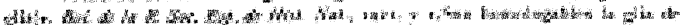

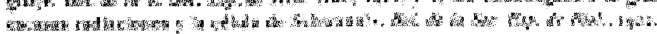

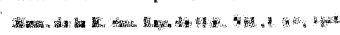

citológicas y su distribución en el neuroeje, sino que en su ultimo trabajo ${ }^{16}$ estableció sus relaciones con las neuronas y, especialmente con las fibras mielínicas.. De este modo aclaro sus funciones que serían aportar una envoltura celular a cada una de las fibras que discurren por el neuroeje . La pseudoneuronofagia dependería, por su parte, del recubrimiento de las neuronas de la sustancia gris, a las que aportaría su vaina citoplásmica. Así, equiparó a la oligodendroglia con las células de Schwann de los nervios periféricos. Los trabajos ultraestructurales posteriores acerca de la célula oligodendroglica y de la microscopía electrónica de la vaina mielínica, no han venido sino a confirmar estos postulados, establecidos muchos años antes. 
FIGURA 10. Imagen del trabajo original «Tercera aportación al conocimiento morfológico e interpretación funcional de la oligodendroglían (1920)

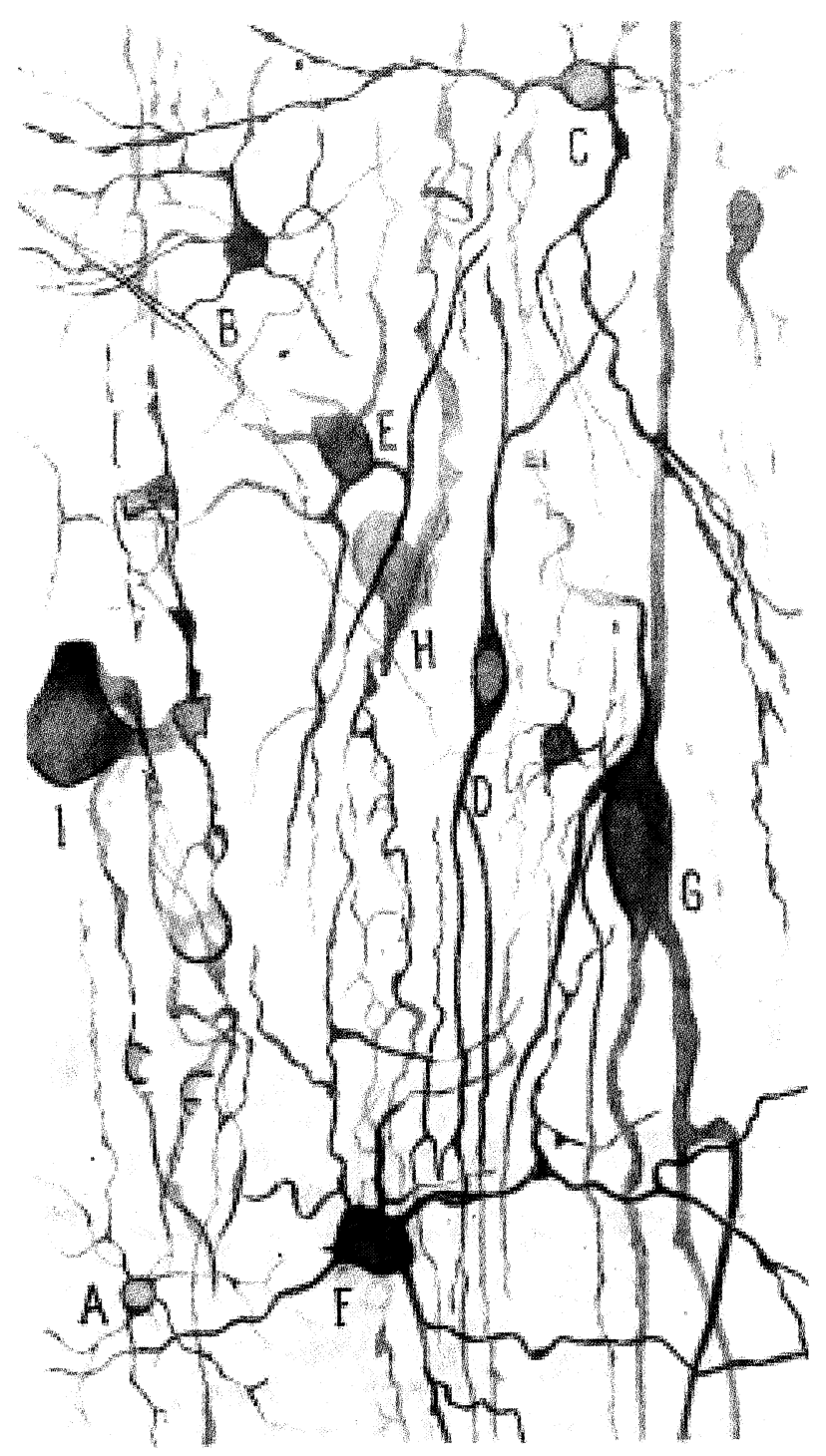

\section{Los tumores cerebrales}

El trabajo básico de Río Hortega fue el «Estudio y Sistematización de los Gliomas y Paragliomas", publicado en 1932, como parte del primer tomo de la revista Archivos Españoles de Oncologia ${ }^{21}$ y que constituyo una de las ponencias al I Congreso Mundial del Cáncer. El libro posterior «Anatomia Microscópica de los Tumores del Sistema Nervioson es una recopilación del anterior sumándole los tumores no gliales $^{22}$.
FIGURA 11. Primera pagina

del original

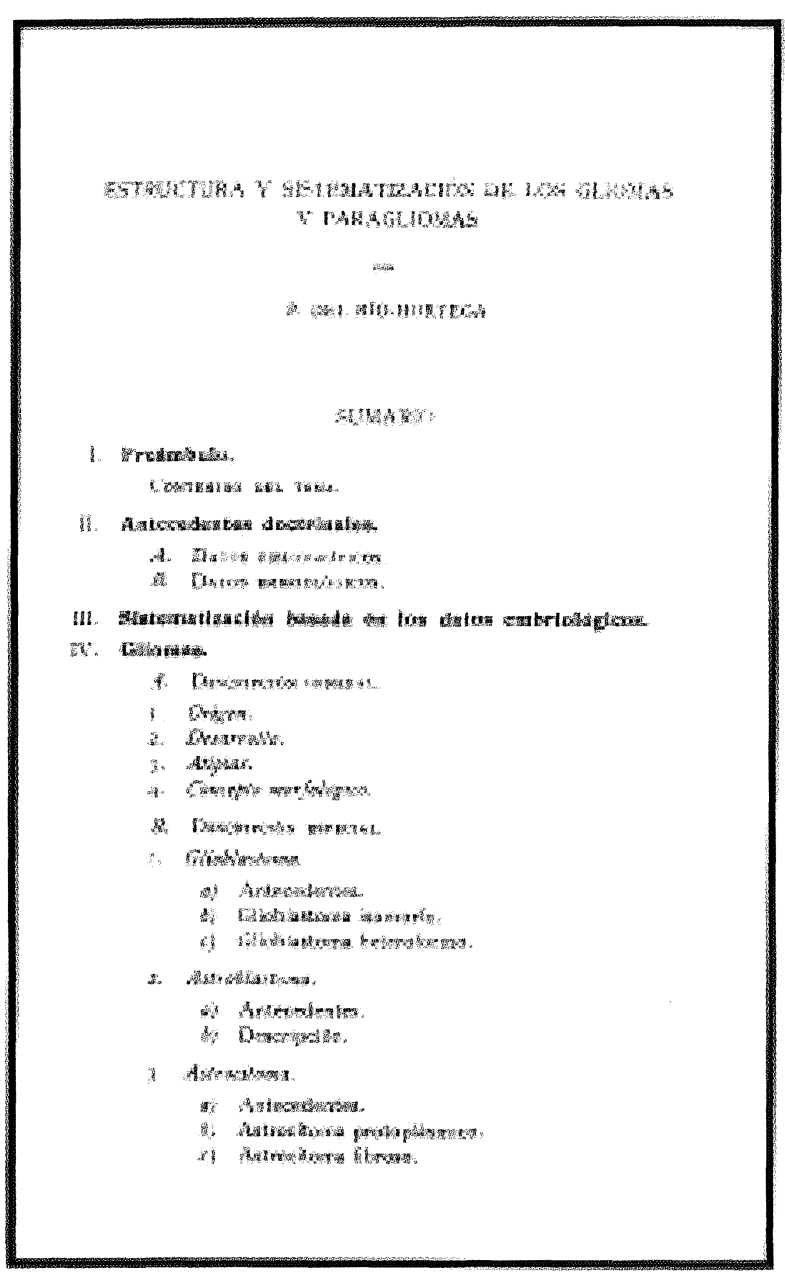

En ambos textos se defiende una clasificación histogenética al estilo de Bailey y Cushing, basándose en el uso exclusivo de las impregnaciones argentinas para establecer un paralelismo entre la evolución citogenéticas de la neuroglia y las imágenes de los tumores cerebrales.

Es evidente que desde la óptica actual, la obra de RíoHortega resulta excesivamente ligada al estudio citológico y que esta basada en un sistema clasificatorio abandonado, careciendo de aplicabilidad clínica, pero no cabe

ARBOR CLXXXI 714 JULIO-AGOSTO (2005) 221-232 ISSN: 0210-1963 
duda de que en su tiempo -73 años antes- fue un hito extraordinario, especialmente en un momento en que la

FigURA 11. Primera pagina del original

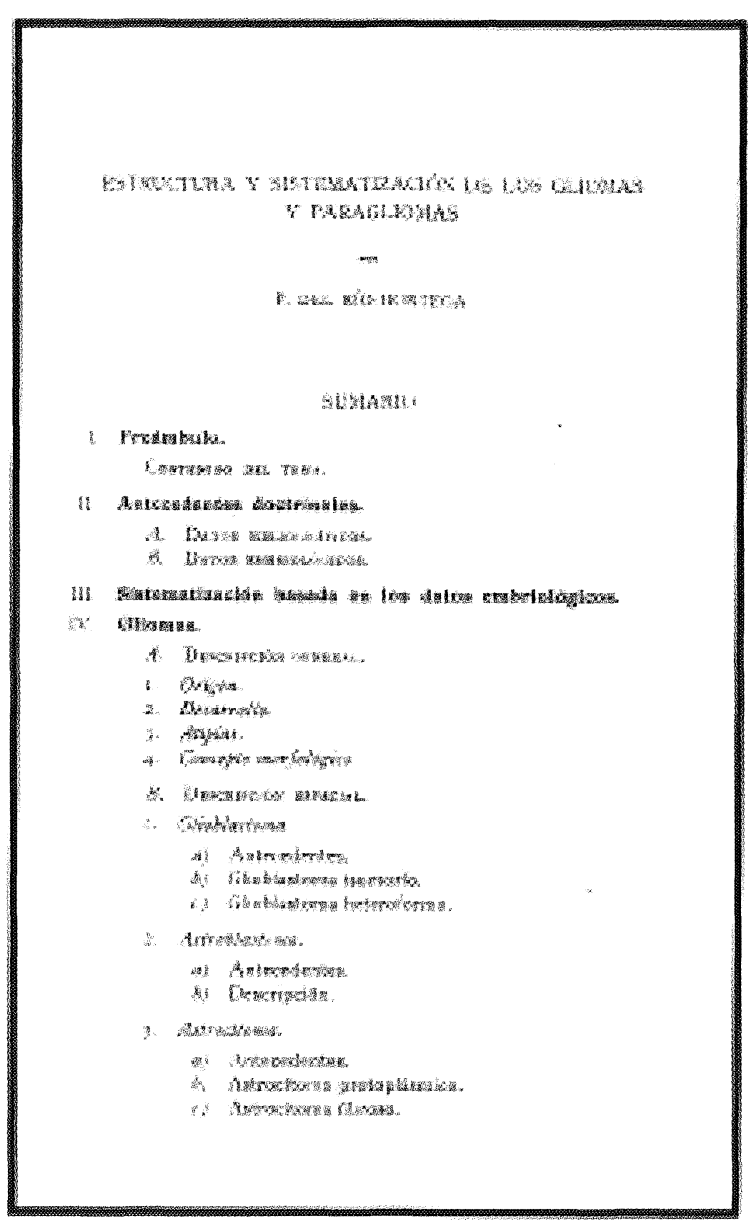

\section{Las consecuencias}

El descubrimiento de los métodos con carbonato de plata y de las dos estirpes celulares citadas, tuvo por consecuencia una serie de aplicaciones, especialmente en el terreno de la patologia, ya que los trabajos de Río Hortega acerca de ambas células habian aclarado casi todo lo concerniente a la morfología en estado normal.

\section{Microglía}

Las consecuencia lógica de la identificación de la microglia como un macrófago adaptado a la textura del Sistema Ner- visualización del citoplasma celular era un empeño imposible.

FiguRA 12. Portada del original

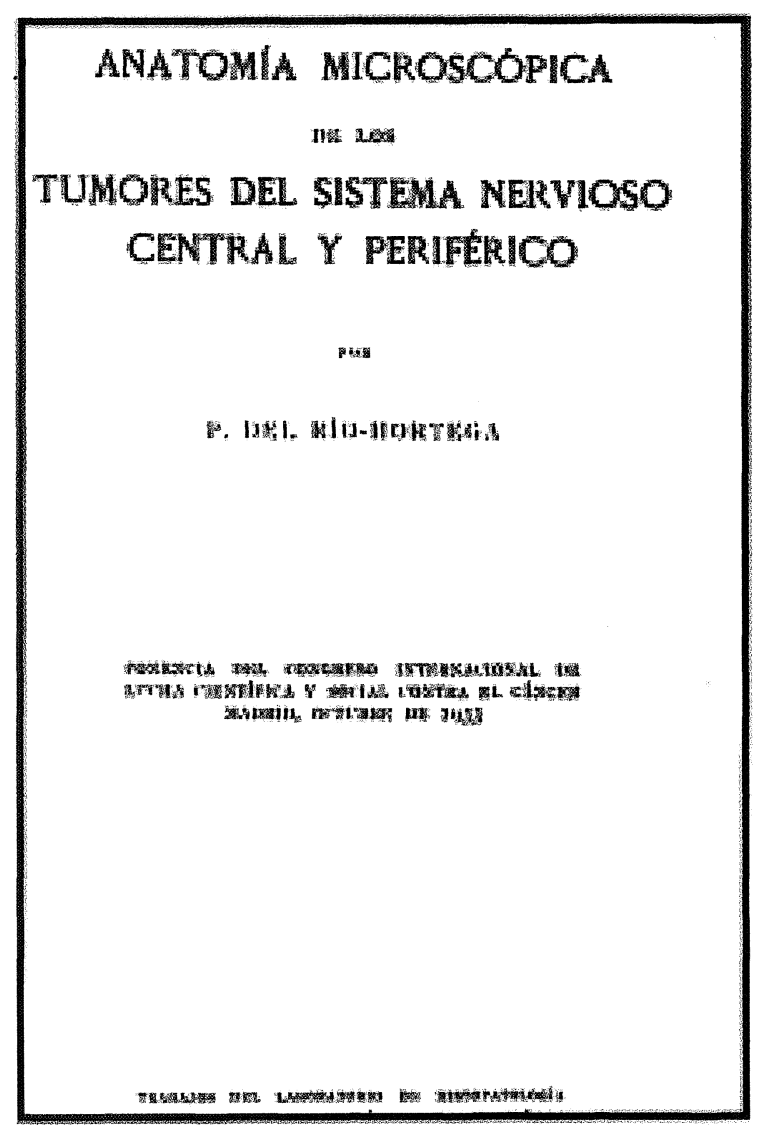

vioso, es su participación en todos los mecanismos propios de esta estirpe celular. De este modo, desde los trabajos de Rio Hortega y Penfield ${ }^{23}$ se conoce el papel de la microglía activada tras la producción experimental de heridas en la corteza cerebral. Inactiva en principio, la microglia se activa como respuesta inflamatoria transformándose sucesivamente en células en bastoncito, células ameboides y, por su capacidad fagocitaría, en corpúsculos granuloadiposos. Esta activación conlleva la traslación de estas células a lo largo de los intersticios del Sistema Nervioso, hasta alcanzar el foco inflamatorio, fase durante la cual tienen lugar las transformaciones citadas. Esta interpretación de la evolución de la microglia, ha permanecido vigente hasta la actualidad. 
No quiere decir esto, que todos los macrófagos del foco inflamatorio sean de origen microglial, pero si que, aunque buena parte arriben al foco por vía sanguínea, un componente importante deriva de células microgliales activadas.

\section{Oligodendroglía}

Una de las consecuencias más llamativas de estos trabajos es la imagen microscópica de los oligodendrogliomas. La llamada imagen en huevo frito, que aparece también en alteraciones no tumorales como el edema cerebral, y su asociación en la imagen en panal de abejas, existen únicamente en material fijado en formol. Las preparaciones en fresco o con fijación idónea en glutaraldehido o las células emigradas en los cultivos de tejido, carecen de este aspecto. Se trata pues de un artefacto por la rápida alteración citoplásmica, bien por alteraciones del medio como en las reacciones oligodendrogliales o por la fragilidad de los oligodendrocitos tumorales.

Consecuencia de los trabajos de Río Hortega es la descripción de dos tipos de oligodendroglioma que difieren de la imagen clásica. Realmente, pocos tumores son tan fáciles de diagnosticar como el oligodendroglioma convencional, pero ya en 1956 , Zülch ${ }^{24}$ señaló que, estudiados con métodos especiales, estos tumores distan mucho de presentar la monótona imagen habitual, apareciendo otros tipos celulares. Subsiguientemente, surgen variantes histológicas que plantean numerosos problemas interpretativos.

De un lado, los tumores de citoplasma eosinófilo y opaco fueron ya entrevistos por Takei y cols $^{25}$ y mas tarde estudiados a fondo por Escalona Zapata ${ }^{26}$, Herpers y Budka ${ }^{27}$ y Meneses y $\operatorname{Kepes}^{28}$. El primer punto en litigio reside el carácter regresivo o progresivo de este tipo celular. Su persistencia a lo largo de la vida del cultivo ${ }^{26}$, la existencia de gliofilamentos en su interior ${ }^{28}$ asi como la expresión de GFAP en los propios cultivos y en gran parte de las células tumorales abogan por su naturaleza progresiva ${ }^{29}$.

De otro, los tumores mixtos u oligodendroastrocitomas ${ }^{30}$ plantean la relación citogenética entre ambas estirpes celulares. Admitida la procedencia común a partir del glioblasto, existen partidarios de un desarrollo tumoral a partir de los lineas distintas y autores adscritos a un origen común en el que algunas células oligodendróglicas serian capaces de desarrollar caracteristicas astrociticas. Sobre estas bases se ha propuesto una metaplasia astrocitica de la célula oli- godendróglica tumoral para explicar la naturaleza del oligodendrocito eosinófilo (minigemistocito) y la de al menos una parte de los tumores mixtos. Los trabajos de Escalona Zapata $^{29}$ demostrando en series paralelas, teñidas con GFAP e impregnaciones argénticas, la progresión astrocitica en los oligodendrogliomas apoyan aún más este punto de vista.

A menudo, estas variantes están en relación con los cuatro tipos descritos por Rio Hortega ${ }^{16}$ que parecen haber sido olvidadas.

En este mismo sentido pesa la opinión de Lumsden que defiende que la gliosis propia de las leucodistrofias, depende de la transformación astrocítica de previos oligodendrocitos, lo que explicaría la escasez de dichas células en estos procesos. Esta opinión refuerza el concepto de metaplasia astrocítica para explicar la expresión de GFAP en tan elevado numero de oligodendrogliomas $y$, por supuesto, la existencia de oligoastrocitomas.

\section{Los tumores cerebrales}

La aportación de Río Hortega al estudio de los tumores del Sistema Nervioso resulta un hecho natural tras la introducción de sus métodos. Así, en este terreno influyó decisivamente en varios campos.

Ya de inicio no debe olvidarse la dedicatoria personal del libro original de Bailey y Cushing ${ }^{31}$ existente en la Biblioteca del Instituto Cajal de Madrid, en la que se citan las técnicas de impregnación argéntica de la Escuela Española como importante base para su publicación. Estas técnicas fueron la impregnación con oro-sublimado de Cajal y los métodos con carbonato de plata de Río Hortega.

La influencia de Río Hortega en este tema se ha reflejado en múltiples publicaciones posteriores.

Así, el capítulo de Folke Henshen en el Handbuch der Spezielle Pathologische Anatomie und Histologie de Henke y Lubarsch de $1954^{32}$ está repleto de de imágenes de impregnación argéntica y del espiritu de Río Hortega.

Igualmente Zülch 24,33 cita los estudios de Río Hortega, aceptando su punto de vista clasificatorio y la división propuesta por el, ya en 1932, en gliomas y paragliomas. 
En 1962 se produce la versión inglesa del libro de Río Hortega acerca de los tumores cerebrales, publicado por la Editorial Charles C. Thomas de Springfield (Illinois) ${ }^{34}$.

Consecuencia también de la influencia de Río Hortega, y en esta caso de su estancia en Oxford junto a Dorothy Russell es la descripción de la llamada microgliomatosis por Russell, Marshall y Smith ${ }^{35}$, gracias a una curiosa variante de impregnación metálica no argéntica. Previamente, autores como Belezky ${ }^{36}$, Awzen $^{37}$, Benedek y Juba ${ }^{38}$ o Abbott y Kernohan ${ }^{39}$ describieron con diversos nombres, neoplasias de este tipo. Incluso Polak 40 defendió el concepto de microglioma a pesar de que el propio Río Hortega negara siempre la existencia de tumores de la microglia. A la luz de los actuales conocimientos, lo mas probable es que, en unos casos se tratara de gliomas malignos con acusado componente microglial por necrosis o linfomas estudiados antes del advenimiento de los métodos inmunohistoquímicos que permitieron su adecuada tipificación. De este modo se volvió a la antigua concepción de Río Hortega: los microgliomas no existen.

Por ultimo, e independientemente de las numerosas citas bibliografícas en los mas importantes libros de Neuropa- tología, no pueden dejar de citarse aquí los libros publicados por seguidores de Río Hortega de distintas nacionalidades. Así el libro de Polak ${ }^{41}$, el libro de Scharenberg y Liss de 42 , la monografía de Costero, Barroso-Mogel y Chevez $^{43}$.

\section{Epílogo}

No queremos terminar esta revisión sin hacer constar que los autores han querido evitar aludir a aspectos históricos de Río Hortega. Se han publicado numerosas revisiones sobre su evolución histórica, su trayectoria sociopolítica y sus avatares personales y a ellas nos remitimos ${ }^{44-49}$. Los autores han querido destacar sus aportaciones a la Histología y a la Anatomía Patológica y las consecuencias que tuvieron en el desarrollo de ambas especialidades, así como lamentar que esta tradición se haya perdido con el paso de los años. No se trata aqui de revivir una metódica que cumplió sus objetivos en su tiempo para, como otras tantas, quedar obsoleta, pero si de echar en falta una generación capaz de actualizar con nuevos métodos una tradición lamentablemente interrumpida. 
1 Río-Hortega, P. Lo que debe entenderse por tercer elemento de los centros nerviosos. Bol. Soc. Esp. Biol. 11, 33-35, 1924.

2 Achucarro,N.- Algunos resultados obtenidos por el procedimiento del tanino y la plata amoniacal. Trab. Lab. Invest. Biol. 9, 269-288, 1911.

3 Ramón y Cajal, S. y De Castro F. Elementos de técnica micrográfica del Sistema Nervioso. Tipografia Artistica. Madrid 1933.

4 Rio-Hortega, P. Noticia de un nuevo y fácil método para la coloración de la neuroglia y del tejido conjuntivo. Trab, Lab. Invest. Biol. 15, 367-378, 1918.

5 Rosai, J. Apología de la hematoxilina y eosina. Rev. Esp. Patol. 32, 499-505 1999

6 Rio-Hortega. P. El método del carbonato de plata. Revisión general de sus técnicas y aplicaciones en histologia normal y patológica. Arch. Hist. Normal y Patol (Buenos Aires) 1,165-205; 329-361. 2, 231-244;577-604. 1945.

7 Robertson, W. A microscopic demonstration of he normal and pathological histology of mesoglia cells. Jour. Mental Sci. $724,1900$.

8 Capobianco. Cit en Rio-Hotega 1928 (16).

9 Hatai. Cit. En Rio-Hortega 1928 (16).

10 Río-Hortega, P. La microglia y su transformación en células en bastoncito y corpúsculos granuloadiposos. Trab. Lab Invest. Biol. 18, 37-83, 1920.

11 Rio-Hortega, P. El tercer elemento de los centros nerviosos: I. La microglia en estado normal. II. Intervención de la microglia en los procesos patológicos. III. Naturaleza probable de la microglia. Bol. Soc. Esp. Biol. 8, 68-82; 8, 91-103; 8, 109-120, 1920.

12 Río-Hortega, P. Histogénesis y evolución normal; éxodo y distribución regional de la microglía. Mem. Real. Soc. Esp. Hist. Nat. 11, 213-268, 1921.

13 Fujita, S. y Kitamura, T. The origin of brain macrophages and the nature of microglia. En Zimmermann: Prog. Neuropath. Vol. III/1.Grune Station N.York 1976.

14 Rio-Hortega, P- Estudios sobre la neuroglia. La glia de escasas radiaciones (oligodendroglia). Bol. Soc. Hist. Nat. 21, 16-43, 1921.
15 Río-Hortega, P. ¿ Son homologables la glía de escasas radiaciones y la célula de Schwann? Bol. Soc. Esp. Biol. 9, 25-28. 1922.

16 Rio-Hortega, P. Tercera aportación al conocimiento morfológico e interpretación funcional de la oligodendroglia. Mem. Real Soc. Esp. Hist. Nat. 14, 161280, 1928.

17 Penfield, W. Oligodendroglia and its relation to classical neuroglia. Brain, 53, 430452, 1924.

18 Metz, A y Spatz. H. Die Hortegasche Zellen (das sogenannte dritte Element) und über ihre funktionelle Bedeutung. Zeitschrf. Neurol Psychiatr. 79, 138-170, 1924.

19 López Enriquez, M. Oligodendroglia de las vías ópticas. Bol. Real. Soc. Esp. Hist. Nat. 26, 1926.

20 Marchesani, 0. Die Morphologie der Glia im Nervus opticus und in der Retina dargestellt nach dem neuesten Untersuchungsmthoden und Untersuchungsergebnissen. Von Graefes Arch. Ophtalm. 117, 575-605 1926.

21 Rio- Hortega, P. Estudio y sistematización de los gliomas y paragliomas. Arch. Esp. Oncol. 2, 411-677, 1932.

22 Rio-Hortega, P. Anatomía microscópica de los tumores del sistema nervioso central y periférico. I Congr. Internat. Cáncer. Madrid 1934

23 Rio-Hortega, P. y Penfield, W. Cerebral cicatrix. Reaction of neuroglia and microglia to brain wounds. Johns Hopkins Hosp. Bull. 41, 278- 1927.

24 Zülch, K.J. Pathologie der raumbeengenden intrakraniellen Prozessen. En TönnisOlivecrona. Handb. Neurochir. Tomo III. Springer. Berlin. 1956.

25 Takei, Y.; Mirra, S.S. y Miles, M.L. Eosinophilic granular cells in oligodendrogliomas. An ultrastructural study. Cáncer 38, 1968-1976, 1968.

26 Escalona Zapata, J. Unusual oligodendrogliomas. Acta Neuropath. Berlin Suppl.VII 94-95, Springer. Berlin 1981.

27 Herpers, M.J.H.M. y Budka, H. Fibrillary Acidic Protein in oligodendroglial tumors, Gliofibrillary oligodendroglioma and transitional oligoastrocytoma as subtypes of oligodendroglioma. Acta Neuropath 64, 265-272, 1984.
28 Meneses, A.C.O. y Kepes, J.J. y Sternberg, N.H. Astrocytic differentiation of neoplastic oligodendrocytes. J. Neuropath. Exper. Neurol. 41, 368,1982.

29 Escalona Zapata, J. Astrocitos en oligodendrogliomas. Estudio con impregnaciones argénticas, inmunohistoquimica y cultivo de tejidos. Rev. Esp. Patol. 35, 533-542, 2002.

30 Hart, M.N.; Petito, C.K. y Earle, K.M. Mixed glioms Cancecr, 33, 134-240, 1974.

31 Bailey, Py Cushing. H. A clasification of the tumors of the glioma group on a histogenetical basis with a correlated study of prognosis. J.B. Lippincott Co. Philadelphia 1926.

32 Henschen, F. Tumoren des Zentralnervensystems und seiner Hüllen. En: HemkeLubarsch: Handb. Spez, Path. Anat. Tomo XIII, Springer. Berlin 1955.

33 Zülch, K.J. Brain Tumors. Their Biology and Pathology. Springer. N.York. 1965.

34 Rio-Hortega, P. The microscopic anatomy of tumors of the central and peripheral nervous system. Charles C. Thomas. Springfiel 1962.

35 Russell, D.S. Marshall, A.H.E. y Smith, F.B. Microgliomatosis $A$ form of reticulosis affecting the brain Brain, 71, 1-15, 1948.

36 Belezky, W.K. Ein Fall von Mesogliom. Virchows Arch. 290, 450-459, 1933.

37 Awzen, A.P. Du type special de tumeurs mesenchymals non muries du system nerveuse central. Acta. Med. Scand. 87, 470, 1936.

38 Benedek, L. y Juba, A. Über das Mikrogliom. Dtsch.Z. Nervenheilk. 152, 159169, 1941.

39 Abbott, K.H. y Kernohan, J.W. Primary sarcomas of the brain. Review of the literature and report of 12 cases. Arch. Neurol. Psychiatr. 50, 43-66,1943.

40 Polak, M. Sobre la histopatologia de los microgliomas cerebrales. Arch. Hist. Normal. Patol. (Buenos Aires) 5, 41,1952.

41 Polak, M. Blastomas del sistema nervioso central y periférico. Patología y ordenación histogenética. López Libreros Ed. Buenos Aires 1966.

42 Scharenberg K y Liss, L. Neuroectodermal tumors of the central and peripheral nervous system. Williams and Wilkins Co. Baltimore. 1969. 
43 Costero, I., Barroso-Moguel, R. y Chevez, A . Biologia de los gliomas. Edamex. México 1979.

44 Obrador, $S$ y otros. Homenaje a Rio-Hortega. En memoria del $X X$ aniversario de su muerte Rev Esp. Oncol 12, 7-52, 1965.

45 Castro, F. de. Cajal y la Escuela Histológica Española. Universidad Complutense. Madrid 1981.

46 Riera J. Pío del Río-Hortega y la ciencia de su tiempo. Acta Histórica-Medica Vallisoletana. Universidad de Valladolid 1994.

47 Izquierdo Rojo J.M. Recuerdo de Pío del Río-Hortega en el $5^{\circ}$ aniversario de su muerte. Neurología, 10,259-276, 1995.

48 Oliva Aldamiz, H. In memoriam. Pío del Rio-Hortega (1882-1945. Rev. Esp. Patol. 32, 507-515,1999.

49 Escalona Zapata, J. Historia de la Anatomia Patológica madrileña. McLine S.L. Madrid 2003. 